\title{
Médecins, mettez un bibliothécaire dans votre moteur (de recherche)!
}

\section{Doctors, put a librarian in your (search) engine!}

\author{
Ludovic HÉRY ${ }^{1,5}$, Catherine WEILL ${ }^{2,5}$, Benjamin MACÉ ${ }^{2}$, David BENOIST ${ }^{2}$, \\ Alexandre BOUTET ${ }^{2}$, Hélène DEFAUX ${ }^{1}$, Claire NGUYEN ${ }^{2,5}$, \\ Nathalie PIÑOL-DOMENECH ${ }^{3}$, Françoise FONTAINE-MARTINELLI ${ }^{3}$, \\ Olivier LEGENDRE ${ }^{4}$ \\ 1 Centre hospitalier universitaire de Clermont-Ferrand, France \\ 2 Bibliothèque interuniversitaire de Santé Paris, France \\ 3 Bibliothèque Clermont Université - BU santé, France \\ 4 Bibliothèque Clermont Université - BU numérique, France \\ 5 Membre du groupe de veille Couperin en Sciences de la vie et de la santé
}

Manuscrit reçu le 22 décembre 2014 ; commentaires éditoriaux formulés aux auteurs le 19 janvier 2016 ; accepté pour publication le 8 mars 2016

Mots-clés
Recherche
documentaire ;
bibliothécaire ;
interne ; médecin;
bases de données
bibliographiques ;
formation ; open
access

Keywords
Information retrieval;
Research skills;
Hospital librarians;
Residents;Physicians;
Bibliographic
databases; Training;
Open Access

Open Access

\begin{abstract}
Résumé - Contexte et problématique : Tous les médecins, quels que soient leur niveau d'expérience ou leurs statuts, internes, médecins hospitaliers, universitaires ou non, ont besoin d'informations et de documentation. Or, dans beaucoup de milieux et notamment en France, peu maîtrisent la recherche documentaire. Passé le premier essai de recherche sur Google et PubMed, beaucoup se découragent en raison du manque de temps et des difficultés qu'ils rencontrent. Exégèse : Des études menées à l'étranger montrent que l'implication active des bibliothécaires au sein même des équipes médicales (réunions matinales de service, revues de morbi-mortalité, etc.) a un effet mesurable et positif : gain de temps, réduction de la durée d'hospitalisation, économies financières. Dans ce but, les bibliothécaires de santé en France ont mis en place des services de renseignement à distance, des formations, des moyens d'accéder facilement à la documentation. Conclusion : Les bibliothécaires et les médecins pourraient trouver de multiples bénéfices en apprenant à travailler ensemble, au bénéfice du patient.
\end{abstract}

Abstract - Background: Residents, physicians, practitioners, academics, whatever their
position or status, all need scientific information. Yet in France at least, many physicians are
not familiar with information research. They try Google and PubMed, but do not take the
time to develop information retrieval skills. Analysis: International studies show that libra-
rians who are actively involved in medical teams (morning department meetings, morbidity
and mortality reviews) have a measurable and positive impact, saving time and money while
cutting down on hospital stays. To do so, French hospital librarians have set up virtual refe-
rence services, training sessions and easy access to online articles. Conclusion: Collabora-
tion should benefit librarians, physicians... and patients!


Qu'ils soient internes, médecins de ville ou hospitaliers, universitaires ou non, tous les professionnels de santé ont besoin d'informations pour exercer. Pour y accéder, ils utilisent différents canaux : Internet, presse professionnelle, discussion avec les collègues, etc. Mais fort peu pensent à pousser la porte d'une bibliothèque médicale. Et pour cause : la bibliothèque est le plus souvent considérée comme un réservoir à manuels ou un endroit calme pour réviser ses cours et préparer les épreuves classantes nationales (ECN).

Le métier de bibliothécaire est mal connu, son expertise peu reconnue. De leur côté, les bibliothécaires, rarement issus des filières scientifiques, ignorent parfois les spécificités du champ médical et hospitalier.

Il est pourtant impératif de rapprocher médecins et bibliothécaires : une meilleure (re)connaissance réciproque des compétences des uns et des attentes des autres pourrait permettre de mieux servir les médecins, donc les patients. Sur ce point, il nous semble que bien des exemples étrangers nous montrent la voie. En s'appuyant sur ces expérimentations réussies, nous plaidons pour la mise en œuvre d'un rapprochement significatif entre médecins et bibliothécaires, notamment dans le contexte particulier de la France, où cette démarche est à peine esquissée.

\section{Bibliothécaire : un métier à connaître}

\section{Qu'est-ce qu'un bibliothécaire de santé ?}

Un bibliothécaire de santé est un bibliothécaire ou un documentaliste qui travaille dans une bibliothèque universitaire en sciences de la santé (BU santé), une bibliothèque de centre hospitalier universitaire (CHU), une bibliothèque hospitalière, ou dans toute bibliothèque ou centre de documentation en lien avec les disciplines médicales (médecine, pharmacie, odontologie, maïeutique, soins infirmiers, etc.).

Ses activités traditionnelles consistent (ou consistaient) à acquérir des livres et des revues, à les signaler dans les catalogues, à les classer et à les mettre à disposition du public.
Comme ses homologues, mais bien davantage et plus rapidement dans le domaine médical qu' ailleurs, le bibliothécaire de santé a vu émerger depuis une vingtaine d'années la documentation numérique ${ }^{[1]}$. Avec l'essor des ressources électroniques, il est passé graduellement d'un métier fondé sur la constitution de collections imprimées à une approche centrée sur l'usager et ses besoins en information. En simplifiant, on peut résumer ainsi l'activité d'un bibliothécaire de santé à l'ère numérique :

- Acquérir : dans un paysage éditorial où l'essentiel des ressources (bouquets de revues, bases de données, livres électroniques) est numérique, et où dominent quelques grands éditeurs, certains bibliothécaires sont devenus de véritables professionnels de la négociation commerciale. Les négociations se mènent au sein d'un consortium national, constitué d'un vaste réseau de professionnels de la documentation engagés dans les négociations ;

- Donner accès : pour faciliter l'accès aux ressources en ligne, les bibliothécaires conçoivent, avec l'aide de sociétés de service, des sites web et des portails de recherche spécialisés. Dans une bibliothèque, le webmestre est souvent un bibliothécaire ;

- Former : pour être bien utilisés, les outils numériques nécessitent au minimum une petite prise en main, parfois une véritable formation. Les bibliothécaires organisent ces formations et acquièrent ce faisant des compétences pédagogiques, en animant des séances ou en élaborant des supports (cours, web tutoriels, etc.);

- Communiquer : nombre de bibliothèques ont aujourd'hui investi les réseaux sociaux pour dialoguer avec leur public : blog, page Facebook, compte Twitter, mise en valeur d'images via Flickr ou Pinterest, groupes professionnels sur LinkedIn ou Viadeo $^{[2,3]}$.

- Depuis peu, émergent de nouvelles fonctions relatives aux besoins des institutions en expertise bibliométrique, au développement de l'open access, ou à l'impératif naissant de gestion des données de la recherche ${ }^{[4]}$.

Le bibliothécaire doit donc être à la fois spécialisé (dans une discipline, une fonction) et se montrer polyvalent sur le plan technique, dans un dialogue constant avec ses usagers. Or en France, le principal 
organisme de formation des bibliothèques, l'École nationale supérieure des sciences de l'information et des bibliothèques (ENSSIB), recrute essentiellement des étudiants de lettres et sciences humaines. La formation y est généraliste. Les bibliothécaires n'ont donc aucune compétence a priori en médecine. Ils l'acquièrent sur le terrain, au contact des usagers ou par la formation continue.

La notion émergente d'informationiste ${ }^{[5]}$ correspond à cette double compétence : par opposition au bibliothécaire de santé (clinical librarian), l'informationiste (informationist) est en principe un bibliothécaire ayant une formation médicale ou un médecin ayant une formation de bibliothécaire. Il est, de plus, parfaitement intégré à l'équipe médicale ${ }^{[6]}$. Hélas, cet être hybride n'existe guère, ni aux États-Unis ni ailleurs, et constitue pour l'instant un idéal inaccessible. En attendant son avènement, deux des missions exercées par les bibliothécaires mériteraient d'être mieux connues des médecins : la formation à la mâ̂trise de l'information et les acquisitions de documentation électronique.

\section{La formation à la recherche documentaire}

La facilité apparente de l'accès à l'information n'importe quelle recherche dans Google donne des milliers de réponses - masque en réalité la difficulté à trouver une information pertinente et adaptée. S'orienter dans cette profusion requiert une bonne maîtrise des outils de recherche, couplée à une mâ̂trise minimale de la discipline, nécessaire pour formuler les questions avec des termes justes et avoir une vision critique des résultats. La maîtrise de ces outils est le métier des bibliothécaires.

Les bibliothécaires de santé peuvent aider rapidement un médecin à atteindre un excellent niveau de maîtrise des principales bases de données médicales et biomédicales (PubMed en premier lieu, mais aussi le CISMeF, la Cochrane Library...), des outils de recherche et de veille documentaire, et des logiciels de gestion de références bibliographiques.

Bien que les bases de données comme PubMed aient beaucoup évolué dans le sens d'une plus grande facilité d'utilisation, elles demeurent complexes.
Leurs fonctions « avancées », souvent ignorées, sont pourtant nécessaires pour une recherche rapide, fine et précise : faute d'en connaître le maniement, on perd beaucoup en qualité de réponse. Quiconque a déjà comparé, dans PubMed, une recherche en langage libre (« à la Google ») avec une recherche à l' aide du vocabulaire $\mathrm{MeSH}$, sait à quel point les résultats sont différents. En témoigne par exemple le blog de Philippe Eveillard ${ }^{[7]}$, qui donne à ses collègues médecins des conseils pour mieux utiliser PubMed. Une précédente contribution parue dans la revue avait du reste déjà apporté aux lecteurs de précieuses clarifications ${ }^{[8]}$.

\section{L'acquisition de ressources électroniques}

L'accès aux ressources documentaires en santé (revues, bases de données) coûte très cher, surtout depuis qu'elles sont disponibles en ligne. En 2013, le prix moyen d'une revue était de 2653 \$ en biologie, $1482 \$$ en médecine ${ }^{[9]}$. L'inflation annuelle est de $7 \%$ en moyenne ${ }^{[10]}$, bien au-delà de l'inflation générale, pour des plates-formes dont l'ergonomie est par ailleurs très inégale.

Pour combattre cette tendance inflationniste et améliorer le service, les établissements scientifiques français se sont regroupés en un consortium d'achat, Couperin (http://www.couperin.org/), dont les négociateurs sont généralement des professionnels de bibliothèques. En 2013 plusieurs CHU, le Centre national de la recherche scientifique (CNRS) et l'Institut national de la santé et de la recherche médicale (INSERM) ont rejoint le consortium, fort aujourd'hui de plus de 250 membres. En 2013, les professionnels du pôle «sciences de la vie et de la santé » de Couperin ont négocié pour la collectivité une trentaine de ressources : revues Masson, JAMA et AMA, BMJ, NEJM, Nature, PNAS, UpToDate, etc.

Depuis peu, l'Initiative d'excellence en information scientifique et technique (ISTEX : http:// www.istex.fr/), portée par le Ministère de l'enseignement supérieur et de la recherche, donne aux étudiants et chercheurs de tous les établissements académiques (y compris les hôpitaux publics et la Bibliothèque nationale de France) un accès à des archives de revues 
et des livres acquis en licence nationale. Ainsi, depuis 2011 ont été achetées les archives des revues Springer, celles de la Freedom Collection d'Elsevier et huit revues publiées par l'éditeur de Nature.

Ces efforts ne constituent qu'un embryon de réponse au gigantesque problème que pose l'inégalité des accès à l'information scientifique et technique : quel que soit son statut (étudiant, chercheur, praticien), une personne sans rattachement académique (université, laboratoire, CHU) n'a accès à aucune ressource, à moins de s'abonner individuellement. Les périodiques électroniques ne sont accessibles qu'aux membres des institutions abonnées : université, INSERM, CNRS et certains hôpitaux (Assistance Publique - Hôpitaux de Paris, Hospices civils de Lyon, etc.).

La transition vers l'open access, la libre diffusion de l'information scientifique et technique, ne se fera pas en un jour. Elle représente un enjeu majeur pour la recherche et la pratique médicales, et une préoccupation partagée des chercheurs et des bibliothécaires.

\section{Médecins : des besoins à prendre en compte}

\section{Connaître les besoins des médecins}

En France, il existe peu d'études consacrées aux usages des médecins en matière de documentation. De telles études permettraient pourtant de savoir comment les médecins travaillent, quelles bases de données ils utilisent, comment ils y accèdent et à quel moment de la journée, quelle connaissance ils ont des services offerts par les bibliothèques et si ceux-ci correspondent à leurs besoin.

Faute d'études françaises, les études menées à l'étranger peuvent s'avérer très instructives. En 2011, le Research Information Network, un groupe de recherche britannique en sciences de l'information, a mené une enquête auprès des chercheurs du Royaume-Uni ${ }^{[11]}$ pour connaître leurs habitudes de recherche d'information et de lecture des articles scientifiques. Certaines pratiques se révèlent sans surprise : les chercheurs lisent en semaine aux heures de bureau mais aussi la nuit et le week-end. D'autres, en revanche, sont éclairantes pour les bibliothécaires : pour faire leurs recherches, les chercheurs utilisent Google, Google Scholar, le Web of Knowledge et PubMed, en raison de leur simplicité d'utilisation et de leur richesse. Ils ne se servent des portails d'éditeurs (par exemple Science Direct d'Elsevier) que pour télécharger les articles.

Une étude canadienne ${ }^{[12]}$ a permis d'identifier les quatre ressources les plus consultées par les internes et les médecins : les revues électroniques (46\%), PubMed/MEDLINE (42\%), UpToDate $(40 \%)$ et les livres électroniques (30\%). Une étude comparable serait à mener en France. On peut supposer qu'elle donnerait des résultats sensiblement différents : la base UpToDate, par exemple, serait probablement absente du palmarès français.

\section{Être formé à la recherche d'information}

En dressant un rapide état des lieux des formations organisées par les bibliothèques de santé, on constate que celles-ci attirent principalement un public d'étudiants motivés par la préparation d'un mémoire ou une thèse.

Le tronc commun du deuxième cycle des études de médecine comporte une unité d'enseignement de formation générale à la recherche dont l'un des objectifs est d' " apprendre à mener une recherche documentaire sur un sujet ${ }^{[13]}$. La mise en place de l'épreuve de lecture critique d'article dans le cadre des ECN devait également familiariser les étudiants en médecine, futurs internes et médecins, avec la recherche documentaire. On constate généralement, quand ils entament la rédaction de leur thèse, que cette familiarité est loin d'être acquise.

Pourtant, les occasions de pratiquer la recherche documentaire ne manquent pas : durant l'internat, les seniors demandent aux internes de faire des exposés, des cours ou d'écrire des articles qui exigent une revue de la littérature. Mais le premier réflexe des étudiants est d'interroger le moteur de recherche Google, inadapté pour une recherche poussée de documentation scientifique. 
Dans un sondage effectué sur les trois promotions de médecine générale (2009, 2010 et 2011, 53 réponses) à la faculté de médecine de Clermont-Ferrand, on constate que $98 \%$ des répondants connaissent $\mathrm{Pub}$ Med, mais que seulement $35 \%$ l'utilisent et $64 \%$ déclarent vouloir être formés ; $19 \%$ d'entre eux connaissent UpToDate, mais $7 \%$ l'utilisent et $62 \%$ veulent être formés.

Une forte demande de formation aux outils documentaires existe donc chez les internes. Un frein au développement de ces formations peut résider dans l'attitude des encadrants et des professionnels déjà en poste : les enseignants chercheurs et le personnel hospitalier sont eux-mêmes peu nombreux à suivre les formations proposées par les bibliothécaires ${ }^{[14]}$. Cités par Marie Boissière dans son mémoire de diplôme de conservateur de bibliothèque, des professeurs estiment que « les formations documentaires dispensées par la BU visent plutôt les étudiants, et que la formation des chercheurs correspond plus à « une sorte de compagnonnage où ils n'ont donc pas besoin des BU » ${ }^{[15]}$.

Si tel est le cas, il faut sans doute que les bibliothécaires imaginent de nouvelles formes d'accompagnement : au plus près des professionnels au sein des services, à la demande d'une personne ou d'une équipe sur un point particulier, à distance, en horaire décalé, etc.

\section{Gagner du temps}

Le professionnel de santé, confronté à une charge de travail importante et à des décisions cliniques à prendre rapidement pour le bien de son patient, court après le temps. Or le bibliothécaire est en mesure de lui en faire gagner!

Selon Davidoff ${ }^{[5]}$, faire une recherche puis sélectionner l'information pertinente pour résoudre un problème clinique prend une heure, voire davantage. C'est pourquoi les urgences de l'hôpital de Leicester (Angleterre) ont mis en place une collaboration systématique entre urgentistes et bibliothécaires ${ }^{[16]}$ : les bibliothécaires effectuent les recherches à la demande des médecins, au moment où ceux-ci en ont besoin, et leur fournissent les articles pertinents.
Une expérience similaire a été menée à l'hôpital de Harrisburg aux États-Unis ${ }^{[16]}$ : les bibliothécaires participent aux réunions de service, pendant lesquels ils font les recherches nécessaires à la résolution des cas cliniques présentés, répondant aux questions cliniques en direct.

Marie Källberg, bibliothécaire en chef de l'hôpital Karolinska de Stockholm, fait du gain de temps l'argument principal de sa tribune ${ }^{[18]}$. Recherches bibliographiques à la demande, accès aux articles en ligne, aide à la préparation d' exposés, congrès, projets de recherche, aide à la bibliométrie (facteurs d'impact, h-index), toutes ces compétences sont mises en œuvre pour faire gagner du temps aux praticiens qui ne manipulent pas aisément ces outils.

\section{Bibliothécaire, que peux-tu faire pour le médecin?}

\section{Aider à trouver une information de qualité}

On sait que les données publiées dans la littérature sont cruciales dans la prise en charge d'un patient. Déjà citée, l'étude de Bartlett et Marshall ${ }^{[12]}$ a montré que l'exploitation des publications a permis aux médecins de gérer la situation clinique de façon différente pour $71 \%$ d'entre eux. Dans ces cas, le diagnostic et la prescription ont été confirmés ou parfois modifiés, et des malentendus concernant la compréhension de leur maladie par les patients ont pu être évités.

Nous savons que les réponses apportées à des questions posées en soins primaires ont changé la prise en charge clinique pour $60 \%{ }^{[19]}$. Comment aider les médecins à développer davantage d'EvidenceBased Practice? C'est la question que se sont posée les bibliothécaires de santé lors de leur $10^{\mathrm{e}}$ congrès international à Brisbane en Australie ${ }^{[20]}$. La réponse tient, d'après eux, aux efforts convergents des bibliothécaires, de l'institution, des médecins et de la formation.

L'utilité d'un bibliothécaire au plus près des besoins, c'est-à-dire non seulement dans les locaux de la bibliothèque ou à distance sur un ordinateur 


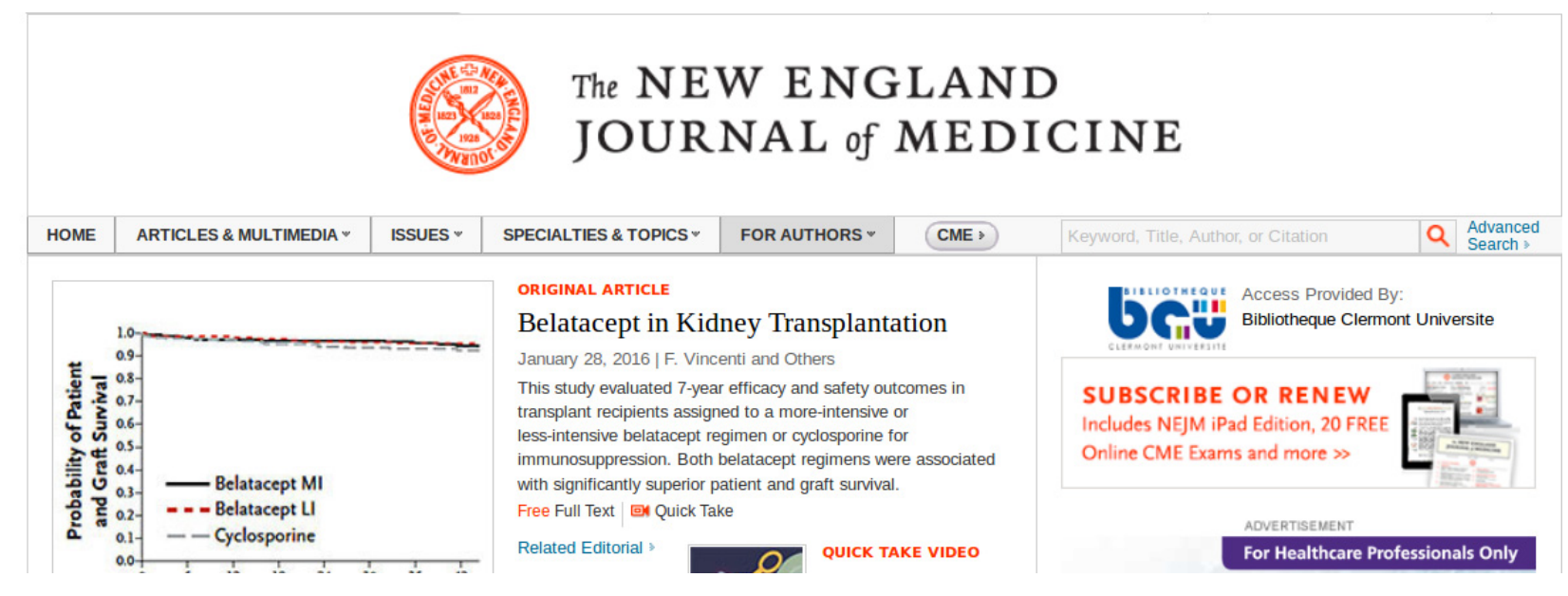

Fig. 1. Accès du New England Journal of Medicine via l'interface universitaire, avec le logo de la bibliothèque universitaire

(recherches bibliographiques, formation, accès au texte intégral), mais également au sein des services hospitaliers, constitue probablement une spécificité du domaine médical. Les expériences en ce sens sont nombreuses dans les pays anglo-saxons ${ }^{[21]}$. Une équipe de chirurgiens de Toronto s'est adjoint les services d'un clinical librarian de janvier à février $2005^{[22]}$ dans le cadre de revues de morbi-mortalité (RMM). Cette expérience a montré des résultats concernant surtout les residents (internes) : élévation du niveau de qualité des recherches et des RMM, recours plus systématique au bibliothécaire pour les recherches documentaires. Celui-ci a même été sollicité après le test : il a participé aux présentations nécessitant une bibliographie de haute qualité (revues de la littérature), à la constitution de bibliographies d'articles, à la préparation de congrès.

En Italie, dans le service de pédiatrie de l'hôpital universitaire de Catanzaro ${ }^{[23]}$, une étude randomisée portant sur 18 internes de pédiatrie a montré le rôle du professionnel de l'information dans la recherche et la sélection d'informations pour la prise de décision clinique. Neuf internes ont été assistés par un bibliothécaire de santé lors de leurs recherches dans $P u b$ Med et d'autres ressources, neuf autres ont travaillé sans aide. Tous ont été confrontés aux mêmes recherches, sur des scénarios cliniques tirés de faits réels. La conclusion de cette étude est que le groupe assisté d'un bibliothécaire est plus performant dans le processus de recherche en ligne avec une différence significative.

\section{Simplifier l'accès aux documents}

Les médecins ont besoin d'accéder d'une façon simple et rapide au texte intégral des documents. Prenons l'exemple d'une bibliothèque universitaire de santé, à Clermont-Ferrand. Lorsque l'on se rend directement sur la page d'accueil du New England Journal of Medicine depuis un ordinateur situé hors campus, une identification est demandée. Mais si la même recherche est effectuée à partir d'un ordinateur du campus, l'accès est direct, un logo avertissant que l'utilisateur est reconnu comme membre de son institution (figure 1).

Dès qu'ils sont hors campus, les habitués utilisent l'accès nomade. Faites le test : utilisez votre moteur de recherche favori pour vous rendre sur la page d'accueil du NEJM : il n'y a pas moyen de consulter la plupart des articles. Si en revanche vous utilisez pour ce faire le lien fourni par votre bibliothèque, il vous demandera de vous authentifier, puis vous accéderez aux articles, où que vous soyez. L'obsession des bibliothécaires est de rendre l'authentification facile, rapide, et même invisible dans certain cas, afin de faire gagner le maximum de temps aux utilisateurs. Les deux procédés ci-dessus sont en général utilisés conjointement, dans ce but : grâce au premier, on 
épargne aux utilisateurs l'étape de l'identification quand ils sont sur site. Grâce au second, la bibliothèque simplifie la procédure d'identification depuis l'extérieur : grâce à un travail effectué par la bibliothèque sur la syntaxe des liens d'accès, la fenêtre d'identification est toujours la même, c'est celle de votre institution.

L'outil numérique donne une impression d'immédiateté et d'accessibilité totale et universelle ${ }^{[24]}$. La réalité est moins miraculeuse : on paie cher, très cher, l'accès aux ressources électroniques. L'accès payant exige que l'utilisateur soit reconnu comme affilié à l'institution dont il dépend, et qui paie les abonnements. C'est pourquoi tout n'est pas accessible à tous tant que l'open access n'est pas devenu la norme.

\section{Proposer des services personnalisés}

Les bibliothèques universitaires françaises ont mis en place depuis une dizaine d'années des services de questions-réponses en ligne, sur le modèle du Ask a Librarian anglo-saxon (http://www.loc.gov/rr/ askalib/). Ces services reposent sur le mail, sur des formulaires, du chat, des sms, de la vidéoconférence, etc. Ils sont gratuits et la plupart du temps ouverts à tous. Certains sont même spécialisés dans le domaine médical, comme le service en ligne des Hospices civils de Lyon (http://portaildoc.univ-lyon1.fr/mabu-en-ligne/demander-un-renseignement/) ou BIUMInfo à la Bibliothèque interuniversitaire de santé (http://www2.biusante.parisdescartes.fr/biuminfo/ biuminfo_sais_fra.htm).

Les réponses apportées sont personnalisées, élaborées à partir de sources multiples et éclairées par un regard critique. Pour les questions complexes, la réponse est généralement différée et envoyée par mail, tandis que le chat, en temps réel, permet d'orienter le lecteur, de donner une réponse de premier niveau ou d'aider à l'utilisation d'outils. L'objectif est d'apporter des réponses appropriées le plus rapidement possible. À l'étranger, Chasing the Sun (http:// salus.sa.gov.au/cts), grâce à une collaboration entre bibliothèques anglaises et australiennes, permet de répondre, quasiment $24 \mathrm{~h} / 24$, aux questions des praticiens et cliniciens.
Les bibliothèques proposent aussi des rendez-vous personnalisés, pendant lesquels un bibliothécaire assiste un médecin, en tête à tête. L'objectif premier est souvent de répondre à une question ponctuelle mais la rencontre permet toujours au praticien de glaner quelques clefs pour une meilleure efficacité : on discute méthode de recherche, typologie des sources, fonctionnalités avancées des outils. Ces rendez-vous, fixés à la demande, répondent à un besoin précis, et s'adaptent aux compétences et à la disponibilité du demandeur bien mieux qu'une formation en groupe.

Il faut probablement pousser plus loin les réflexions pour améliorer ces services, en s'inspirant des exemples réussis à l'étranger. Un usage plus général du chat ou de la visioconférence, pour mener la recherche avec l'usager au moment où il en a besoin, serait notamment une piste à explorer.

\section{Faire économiser de l'argent à la communauté}

Le gain de temps pour les médecins peut se doubler d'un gain financier. Dans une étude menée à l'hôpital universitaire de la Louisiana State University $^{[25]}$, les auteurs ont montré qu'en associant un bibliothécaire à la réunion de service du matin, la durée moyenne de séjour était moindre (la différence entre le groupe associant un bibliothécaire et le groupe témoin est significative). Le coût médian de l'hospitalisation était de 7045 \$ pour le groupe associant un bibliothécaire, contre $10663 \$$ pour le groupe contrôle. Le taux de ré-hospitalisation à 30 jours était identique dans les deux groupes.

Les bibliothèques australiennes ont mesuré le retour sur investissement de leurs services : temps gagné par les médecins, économies réalisées (les informations fournies étant délivrées gratuitement par la bibliothèque). Le résultat est éclairant : le retour sur investissement est de 9 \$ pour 1 \$ dépensé ${ }^{[26]}$.

\section{Mettre à profit les outils du Web}

Mues par le souci de rester proches de leurs utilisateurs et par leur intérêt pour la diffusion du savoir, aiguillonnées par leurs relations tendues avec les éditeurs scientifiques, les bibliothèques ont développé 
une sensibilité et une compétence particulière concernant les outils de partage disponibles sur le web. Les outils de communication du Web social (Facebook, Twitter, etc.) leur sont familiers, de même d'ailleurs que les appareils connectés : de nombreuses bibliothèques expérimentent à ce sujet ou étudient la façon dont leurs usagers travaillent avec les appareils connectés, ou travaillent à identifier les applications utiles pour les médecins ${ }^{[27]}$. Dans un avenir proche, les bibliothécaires pourraient mobiliser leurs compétences en la matière pour aider à suivre certaines épidémies en direct sur la toile ${ }^{[28]}$.

Pour rester dans des enjeux scientifiques, les bibliothèques se sont rapidement familiarisées avec les réseaux sociaux de la recherche tels que ResearchGate et Academia (http://www.researchgate.net/ ; http://www.academia.edu/) et savent en exposer succinctement les mérites et les risques. Elles initient volontiers les chercheurs aux outils de gestion de références bibliographiques, qui procurent un gain de temps considérable dans la rédaction d'articles et la gestion au long cours, voire le partage d'une bibliographie de recherche. Elles peuvent les aider à mettre en place une veille en ligne sur des sujets particuliers, via les réseaux sociaux ou par des moyens plus traditionnels (alertes par courriel, etc. ${ }^{[28]}$. Impliquées dans le développement du libre accès, elles cherchent à faciliter pour les chercheurs l'usage, pour la diffusion de leurs travaux publiés, de dépôt d'archives ouvertes comme HAL : elles s'efforcent d'en démontrer l'intérêt, débrouillent les questions juridiques qui suscitent beaucoup d'appréhensions, proposent une aide à la prise en main voire prennent en charge une partie du dépôt (notamment rétrospectif) pour lancer le mouvement de l'archivage.

\section{La « lettre au Père Noël » : les collaborations à inventer}

En s'inspirant des expériences réussies à l'étranger, nous lançons ici quelques propositions de services innovants de la part des bibliothécaires de santé à destination des médecins :

- Séances de formation ou d'accompagnement, personnalisées et à distance (par webcam, avec partage d'écran), à des horaires adaptés aux besoins des praticiens

- Participation de bibliothécaires aux staffs hospitaliers

- «Faites la recherche à ma place » (le bibliothécaire s'occupe de tout)

- « Trouvez-moi tout de suite cet article/cet ouvrage !»

Vous avez envie d'autre chose qui n'est pas dans la liste ? Écrivez-nous !

\section{Conclusion}

Les bibliothécaires de santé peuvent répondre au double défi de maîtrise de l'information et de gain de temps, en optimisant veille et recherche documentaire au service du praticien. Les bibliothèques proposent déjà de nombreux services susceptibles d'aider les médecins dans leur travail.

Il reste pourtant beaucoup à faire, en France notamment. Tout converge en faveur d'un travail conjoint entre bibliothécaires et praticiens et, pourtant, rares sont les collaborations effectives sur le terrain. Les bibliothécaires doivent sortir de la bibliothèque et les médecins ouvrir la porte de leur service ! Apprenons à travailler ensemble pour le bien des patients.

Et vous, comment s'appelle votre bibliothécaire de santé ? Échangez avec lui (ou elle) comme nous, médecins et bibliothécaires, l'avons fait pour rédiger cet article, et quotidiennement depuis sept ans. Nous ne venons pas du même monde : les bibliothécaires sont des littéraires, ils parlent bibliométrie, coût, HAL, open access, ISTEX, etc. Les médecins sont des scientifiques, ils parlent objectif principal, secondaire, les biostatistiques sont leur niveau de preuve. Mais les uns (les bibliothécaires) sont au service des autres (les médecins) pour les aider à exercer au mieux leur métier.

Changeons de culture. Travaillons ensemble dès que nécessaire. Menons des études communes sur la recherche documentaire (formation des usagers, quels outils, comment, etc.) pour que les bibliothèques offrent des services adaptés aux besoins des médecins. 
Et faisons nôtre sans réserve cette affirmation (sans doute apocryphe mais qu'importe!) d'Albert Einstein : «La seule chose que vous devez absolument connaître, c'est l'emplacement de la bibliothèque »!

\section{Contributions}

Tous les auteurs ont participé solidairement à la rédaction de cet article.

\section{Déclaration d'intérêts}

Aucun auteur ne déclare de conflit d'intérêts en lien avec le contenu de cet article.

\section{Approbation éthique}

Non sollicitée car sans objet.

\section{Références}

1. Carbone P, Cavalier F (Eds.). Les collections électroniques, une nouvelle politique documentaire. Paris : Cercle de la librairie, 2009.

2. Cuddy C, Graham J, Morton-Owens EG. Implementing Twitter in a health sciences library. Med Ref Serv Q 2010;29:32030.

3. Landau R. Solo librarian and outreach to hospital staff using Web 2.0 technologies. Med Ref Serv Q 2010;29:7584.

4. Gaillard R. De l'Open data à l'Open research data : quelle(s) politique(s) pour les données de recherche? 2014 [On-line] Disponible sur : http://www.enssib.fr/bibliotheque-numerique/notices/64131-de-1open-data-a-l-open-research-data-quelles-politiques-pour-les-donnees-de-recherche

5. Davidoff F, Florance V. The informationist: a new health profession? Ann Intern Med 2000;132:9968.

6. Robison RR, Ryan ME, Cooper ID. Inquiring informationists: a qualitative exploration of our role. Evid Based Libr Inf Pract 2009;4:416.
7. Eveillard P. Le blog de phe [On-line]. Disponible sur : http://docedu.fr/WordPress/

8. Broclain D. Utiliser les bases bibliographiques biocliniques à bon escient (Medline, Embase, etc.). Pédagogie Médicale 2004;5:52-60.

9. The winds of change : periodicals price survey. Library Journal 2013 [On-line] Disponible sur : http://lj.libraryjournal.com/2013/04/publishing/thewinds-of-change-periodicals-price-survey-2013/

10. Tillery K. 2012 study of subscription prices for scholarly society journals: society journal pricing trends and industry overview. Allen Press, Inc. 2013 [Online] Disponible sur : http://allenpress.com/system/ files/pdfs/library/2012_AP_JPS.pdf

11. Research information network. E-journals: their use, value and impact: final report. 2011 [On-line] Disponible sur : http://www.rin.ac.uk/our-work/ communicating-and-disseminating-research/ejournals-their-use-value-and-impact

12. Bartlett JC, Marshall JG. The value of library and information services in patient care: Canadian results from an international multisite study. J Can Health Libr Assoc 2013;34:13846.

13. Arrêté du 8 avril 2013 relatif au régime des études en vue du premier et du deuxième cycle des études médicales. Journal Officiel de la République Française 2013 [On-line]. Disponible sur : http:// www.legifrance.gouv.fr/affichTexte.do?cidTexte= JORFTEXT000027343762\&fastPos=16\&fastReqId $=1613774500 \&$ categorieLien $=$ cid\&oldAction $=$ rech Texte

14. Barthet E, Church A, Dailland F, Chevillotte S. Le lien discipline et formation à la maîtrise de l'information : l'exemple de la médecine. 2006 [Online] Disponible sur : http://www.enssib.fr/bibliotheque-numerique/documents/826-le-lien-discipline-et-formation-a-la-maitrise-de-1-information-1exemple-de-la-medecine.pdf

15. Boissière M, Leturcq S. La collaboration entre Service commun de documentation, Faculté de médecine et Centre Hospitalier Universitaire autour des ressources documentaires : réalités, enjeux et perspectives. 2013 [On-line]. Disponible sur : http:// www.enssib.fr/bibliotheque-numerique/documents/ 64496-la-collaboration-entre-service-commun-dedocumentation-faculte-de-medecine-et-centre-hos- 
pitalier-universitaire-autour-des-ressources-documentaires-realites-enjeux-et-perspectives.pdf

16. Coats TJ, Sutton S, Vorwerk C, Cooke MW. In an emergency - call the clinical librarian! Emerg Med J 2009;26:3213.

17. Schwing LJ, Coldsmith EE. Librarians as hidden gems in a clinical team. Med Ref Serv Q 2005;24:2939.

18. Källberg M. [The hospital library can save time for the researching physician]. Läkartidningen 2013;110:698.

19. Alper BS, Stevermer JJ, White DS, Ewigman BG. Answering family physicians' clinical questions using electronic medical databases. J Fam Pract 2001;50:9605.

20. Anuradha S, Kruesi L, Eriksson L, Steele M, Harrison T, Lewis M, et al. Evidence-based practice in action: how can your medical librarian help? Evid Based Med 2010;15:36.

21. Dorset R. Outreach services in healthcare libraries: perceptions and impacts. Health Inf Libr J 2014;31:758.

22. Greco E, Englesakis M, Faulkner A, Trojan B, Rotstein LE, Urbach DR. Clinical Librarian Attendance at General Surgery Quality of Care Rounds (Morbidity and Mortality Conference). Surg Innov 2009; 16:2669.

23. Gardois P, Calabrese R, Colombi N, Deplano A, Lingua $\mathrm{C}$, Longo $\mathrm{F}$, et al. Effectiveness of bibliographic searches performed by paediatric residents and interns assisted by librarians. A randomised controlled trial. Health Inf Libr 2011;28:27384.
24. Dumont R. À l'aube d'une nouvelle ère pour les collections. UdeMNouvelles 2013 [On-line]. Disponible sur : http://www.nouvelles.umontreal.ca/ campus/bibliotheques/20131007-a-laube-dune-nouvelle-ere-pour-les-collections.html?utm_source= web\&utm_medium=image\&utm_campaign=chronique-1

25. Banks DE, Shi R, Timm DF, Christopher KA, Duggar DC, Comegys M, et al. Decreased hospital length of stay associated with presentation of cases at morning report with librarian support. J Med Libr Assoc 2007;95:3817.

26. Edwards J, Ritchie A. Worth every cent and more: An independent assessment of the return on investment of health libraries in Australia. Australian Library and Information Association 2013 [On-line]. Disponible sur : http://www.alia.org.au/sites/default/files/ Worth-Every-Cent-and-More-FULL-REPORT.pdf

27. Aungst TD, Clauson KA, Misra S, Lewis TL, Husain I. How to identify, assess and utilise mobile medical applications in clinical practice. Int $\mathrm{J}$ Clin Pract 2014;68:15562.

28. Giustini D. Social media trends for health librarians: a primer on using social media for clinical disease surveillance. J Can Health Libr Assoc 2012;33:924.

29. Karpinski JL. Disconnected in a Connected World. Med Ref Serv Q 2008;27:5372.

Correspondance et offprints : Ludovic Héry : Centre Hospitalier Régional d'Orléans, Service de médecine interne, 14 avenue de l'hôpital, CS 8670945067 Orleans Cedex 2, France.

Mailto : ludovic.hery@chr-orleans.fr 